\title{
Estructuras Mentales que Modelan el Aprendizaje de un Teorema del Álgebra Lineal: Un Estudio de Casos en el Contexto Universitario
}

\author{
Solange Roa-Fuentes ${ }^{(1)}$ y Marcela Parraguez ${ }^{(2)}$ \\ (1) Escuela de Matemáticas, Facultad de Ciencias, Universidad Industrial de Santander, Ciudad \\ Universitaria Carrera 27, Calle 9, A.A. 678 Bucaramanga-Colombia. \\ (2) Instituto de Matemáticas, Facultad de Ciencias, Pontificia Universidad Católica de Valparaíso, Blanco \\ Viel № 596, Cerro Barón, Valparaíso, Chile. (e-mail: sroa@matematicas.uis.edu.co; \\ marcela.parraguez@pucv.cl)
}

Recibido Feb. 1, 2017; Aceptado Mar. 21, 2017; Versión final Abr. 26, 2017, Publicado Ago. 2017

\begin{abstract}
Resumen
Se presenta una investigación cualitativa que indaga sobre cómo estudiantes universitarios chilenos y colombianos aprenden un teorema del álgebra lineal, denominado teorema Transformación Lineal Matricial, TLMA. Para esto se define un modelo cognitivo en términos de las estructuras y mecanismos mentales que propone la teoría APOE (Acción, Proceso, Objeto, Esquema) para interpretar el pensamiento matemático avanzado. Además se describen los niveles de evolución intra, inter y trans del esquema del teorema TLMA y sus indicadores de desarrollo en términos de relaciones, transformaciones e invariantes. Los resultados de la investigación muestran por un lado que la construcción de la representación matricial de una transformación lineal como objeto, es fundamental para que estudiantes universitarios de primer año comprendan el teorema TLMA; y por otro, que dicho objeto debe ser asimilado por el esquema de transformación lineal.
\end{abstract}

\section{Mental Structures that Model the Learning of a Linear Algebra Theorem: A Case Study in the University Context}

\begin{abstract}
In this paper, a qualitative research that explores how Chilean and Colombian university students learn a linear algebra theorem, called Matrix Linear Transformation theorem, MALT, is presented. For this, a cognitive model is defined in terms of the structures and mechanisms proposed by the APOS theory (Action, Process, Object, and Schema) to interpret the advanced mathematical thinking. Furthermore, the levels of Intra, Inter and trans evolution of the MALT theorem and its development indicators in terms of relationships, transformations and invariants are described. The results of this research show on the one hand, that the construction of the matrix representation of a linear transformation as object is essential for first year students to understand the MALT theorem; and on the other hand that such an object must be assimilated by the linear transformation schema.
\end{abstract}

Keywords: TLMA theorem; APOS theory; linear algebra; genetic decomposition; learning 


\section{INTRODUCCIÓN}

En instituciones universitarias de Latinoamérica es posible encontrar cursos de álgebra lineal cuyo desarrollo está determinado por los contenidos temáticos dispuestos en un programa de estudio; en algunos casos se cuenta con más de una propuesta de organización de contenidos y variaciones entre ellos: Clásica, Categórica, Moderna, Matricial, entre otras (http://matematicas.uis.edu.co/formacion/ciclobasico). Los programas pueden incluir elementos concretos, matrices y vectores, y/o abstractos como espacios vectoriales y transformaciones lineales (Carlson et al., 1997). De acuerdo con Dubinsky y McDonald (2001) el estudio del álgebra lineal es el camino hacia el desarrollo del pensamiento matemático avanzado; por tanto un curso con énfasis en la mecanización de operaciones con n-uplas y matrices, desconoce la naturaleza misma del álgebra lineal, su esencia generalizadora y unificadora de conceptos (Dorier, 2000). Un curso de álgebra lineal clásico, inicia generalmente con el estudio de espacios vectoriales. Esto es algo nada común para los estudiantes quienes súbitamente se encuentran con sofisticadas definiciones y teoremas, que incluso deben demostrar. Sin embargo, en algunos casos el uso de los teoremas se reduce a la aplicación mecánica de un resultado. Al respecto, Abramovitz et al. (2009) mencionan: "Los estudiantes piensan que el teorema puede ser memorizado como un "lema", así puede ser fácilmente recuperado de la memoria bajo el efecto hipnótico de un encantamiento mágico. Sin embargo, usar un teorema como encantamiento mágico puede aumentar la tendencia a usarlo descuidadamente sin tener en cuenta la situación o los detalles de su aplicabilidad"

Para los estudiantes de álgebra lineal, que enfrentan por primera vez un curso de esta materia, los teoremas les pueden parecer resultados aislados, desmembrados y no relacionados con sus estructuras matemáticas preexistentes. Particularmente la estructura lógica sobre las cuales se sustentan los teoremas: hipótesis, demostración y tesis, los deja paralizados frente a cuestiones tan primarias como la escritura formal de la demostración de un teorema. Si bien actualmente existe una tendencia a retrasar el momento en que se supone que los estudiantes han de dominar las técnicas de las demostraciones matemáticas formales, varios estudios se han ocupado de los comienzos en la comprensión y en la producción informal de demostraciones (Balachef, 1987). Uhlig (2003) propone que un primer curso de álgebra lineal debería focalizarse para generar la transición hacia las matemáticas formales. Esta transición requiere de la reflexión sobre la enseñanza y del reconocimiento de las ideas previas que sobre la matemática tienen los estudiantes.

El objetivo en este escrito es analizar cómo un estudiante puede comprender un teorema particular de álgebra lineal, que relaciona las transformaciones lineales y matrices mediante las operaciones composición y producto respectivamente; este resultado es llamado en este escrito teorema TLMA. La construcción del teorema TLMA parte de la construcción de relaciones entre estructuras matemáticas y los mecanismos calculatorios que un estudiante ha logrado sistematizar en un andamiaje previo, para ponerlo a prueba en las nuevas situaciones matemáticas que enfrenta. Se postula que la construcción exitosa de teoremas, genera la evolución de las estructuras matemáticas de los estudiantes universitarios. Esto con base en las fuertes relaciones que deben establecer entre conceptos previos y las nuevas relaciones para lograr su comprensión. Lo que implica desde la teoría APOE (acrónimo de Acción, Proceso, Objeto, Esquema) que la construcción de un teorema genera la evolución y asimilación de nuevos Objetos que forman parte del Esquema de conceptos básicos del álgebra lineal, en el que un nuevo resultado se integra. De tal manera que un individuo podrá evocar componentes del Esquema de manera coherente, para dar solución a nuevas situaciones (Arnon et al., 2014). A partir de los postulados planteados se proponen las siguientes preguntas de investigación: ¿Qué estructuras y mecanismos mentales debe mostrar un estudiante universitario para comprender el teorema TLMA? Si la comprensión de teoremas puede propiciar la construcción de Esquemas ¿cómo se define su evolución a través de los niveles de la tríada Intra, Inter y Trans?

Para dar respuesta a las preguntas planteadas se toma como método de la investigación el Ciclo propuesto por APOE que contempla el desarrollo de tres componentes: i) Análisis teórico, ii) Diseño e implementación de enseñanza y iii) Observación, análisis y verificación de datos (Arnon et al., 2014). El desarrollo de la tercera componente se desarrolla como un Estudio de Casos (Stake, 2010), que incluye estudiantes universitarios chilenos y colombianos.

El análisis que se presenta en este documento, ofrece una descripción sobre la comprensión e interpretación a través de estructuras mentales, de un resultado matemático que requiere de varias conexiones entre distintos conceptos fundamentales que componen el álgebra lineal. Particularmente entre las transformaciones lineales y su representación matricial, a través de las operaciones composición de funciones y producto matricial, respectivamente. La descripción de los niveles del Esquema del teorema TLMA se ancla en el concepto de base de un espacio vectorial finito dimensional, que emerge desde una perspectiva específica, como base canónica de un espacio vectorial de dimensión finita, hasta la relación que establece en la construcción de la matriz que representa una transformación lineal. 
Se espera que la problemática presentada en este escrito contribuya a la discusión sobre la evolución del proceso de abstracción en estudiantes universitarios en el contexto de la construcción de Esquemas básicos del álgebra lineal. Esto con la finalidad de brindar a la comunidad espacios de reflexión sobre cómo generar verdaderos procesos de aprendizaje en los estudiantes, a partir de la indagación profunda y sistemática sobre los mecanismos y estructuras mentales involucradas en la construcción de teoremas matemáticos. Como se muestra en el desarrollo del artículo, la Descomposición Genética concebida como un modelo cognitivo de construcción, es una herramienta potente fundamental en la cimentación de cursos universitarios, en particular de álgebra lineal.

\section{UN TEOREMA EN ÁLGEBRA LINEAL: EL TEOREMA TLMA}

El teorema TLMA se encuentra definido de diferentes maneras en los libros de álgebra lineal, en particular en esta investigación se considera la definición de dos textos: Álgebra Lineal (Hoffman y Kunze, 1993) y Learning Linear Algebra with ISETL (Weller et al., 2002). El primer libro se toma como referente por ser parte de los libros de texto que algunos profesores usan en sus cursos de álgebra lineal. El segundo libro fue escrito por un grupo de investigadores llamado Research in Undergraduate Mathematics Education Community (RUMEC por sus siglas en inglés) quienes estudian diferentes tópicos del álgebra lineal desde la perspectiva de la teoría APOE y usan un lenguaje de programación matemático (ISETL) para propiciar el mecanismo de interiorización. Hoffman y Kunze (1993) inician su libro con un capítulo de Ecuaciones Lineales, un segundo capítulo de Espacios Vectoriales y en el tercer capítulo aparecen las Transformaciones Lineales, allí definen el teorema que hemos llamado TLMA.

Teorema TLMA: Sean V, W y Z espacios vectoriales de dimensión finita sobre un cuerpo $\mathrm{F}$; sea $\mathrm{T}$ una transformación lineal de $\mathrm{V}$ en $\mathrm{W}$ y $\mathrm{U}$ una transformación lineal de $\mathrm{W}$ en $\mathrm{Z}$. Si $\beta, \beta^{\prime}$ y $\beta^{\prime \prime}$ son las bases ordenadas de los espacios $\mathrm{V}, \mathrm{W}$ y $\mathrm{Z}$, respectivamente, y si A es la matriz de T respecto al par $\beta$ y $\beta^{\prime}$ y $\mathrm{B}$ es la matriz de $U$ respecto al par $\beta^{\prime}$ y $\beta^{\prime \prime}$, entonces la matriz de la composición UT respecto al par $\beta$, $\beta^{\prime \prime}$ es la matriz producto $\mathrm{C}=\mathrm{BA}$ (Hoffman y Kunze, 1993). Esta versión del teorema contempla el trabajo sobre espacios vectoriales de dimensión finita y la fijación de las bases para cada espacio. En el caso de Weller et al., (2002) en el Capítulo 6 (Matrices), se enuncian dos teoremas que dan paso a las relaciones presentadas por Hoffman y Kunze (1993).

Teorema 1: Sea $M$ una matriz $n \times p$ sobre $K$ y $N$ una matriz $p \times m$ sobre $K$. Sea $x$ un vector en $K^{m}$, aplicando $N$ a este vector y después aplicando $M$ resulta un vector en $K^{p}$, entonces el vector resultante en $K^{n}$ es igual al vector obtenido aplicando $M N$ al vector original en $K^{m}$. Es decir, $M(N x)=(M N) x$ (Weller et al., 2002). Después de enunciar este teorema Weller et al., describen en el Teorema 2, la relación entre la composición de funciones y el producto de matrices.

Teorema 2: Sean $T: K^{p} \rightarrow K^{n}$ y $S: K^{m} \rightarrow K^{p}$ transformaciones lineales. La matriz que representa la transformación lineal $T \circ S$ es igual al producto entre la matriz que representa la transformación $T$ y la matriz que representa la transformación $S$ (Weller et al., 2002). Weller et al. (2002), proponen que los dos resultados propuestos y demostrados en su libro, corresponden a dos visiones del mismo proceso: composición de transformaciones y multiplicación de matrices tomando en cuenta la base ordenada. En la figura 1, se muestran de manera específica las relaciones que este teorema establece: las flechas superiores indican la composición de las transformaciones lineales $S$ y $T$ definidas entre espacios vectoriales de dimensión finita. Las flechas inferiores indican el producto entre las matrices $N$ y $M$ que corresponden a las representaciones matriciales de $S$ y $T$ respectivamente. Las flechas verticales indican el isomorfismo que puede establecerse entre todo espacio vectorial de dimensión finita y su espacio de coordenadas; así, todo vector de cualquier espacio vectorial puede representarse como un arreglo de coordenadas (Weller et al., 2002).

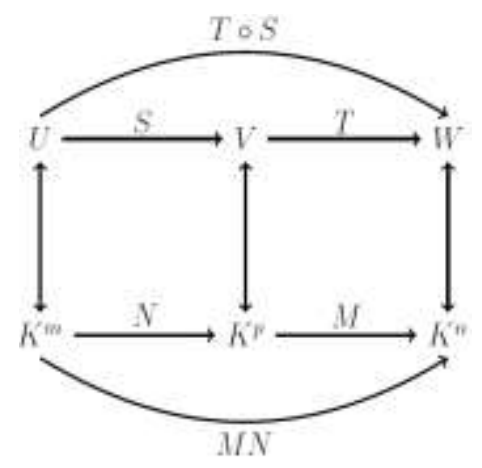

Fig. 1: Relación entre transformaciones lineales definidas entre espacios vectoriales de dimensión finita y su representación matricial (Weller et al., 2002) 
El teorema TLMA es un resultado fundamental para la teoría del álgebra lineal, pues permite identificar la composición de dos transformaciones lineales y construir matrices que se identifiquen con ellas, a partir de coordenadas de vectores, asociados a bases ordenadas específicas de espacios vectoriales dados. Además el teorema TLMA y los conceptos de coordenada y transformación lineal tienen extensiones en otras teorías: Isomorfismos entre espacios vectoriales, el teorema de diagonalización, los vectores propios, entre otros. Otro aspecto importante es el corolario que se desprende del teorema, que muestra cómo actúa la composición de una transformación lineal con ella misma, es decir:

Corolario del Teorema: Sea U un espacio vectorial de dimensión finita sobre K, y B base de U. Si T es una transformación lineal de $U$ en $U$, entonces: $[\mathrm{T} \circ \mathrm{T}]_{\mathrm{B}}^{\mathrm{B}}=[\mathrm{T}]_{\mathrm{B}}^{\mathrm{B}}[\mathrm{T}]_{\mathrm{B}}^{\mathrm{B}}=\left([\mathrm{T}]_{\mathrm{B}}^{\mathrm{B}}\right)^{2}$ (Figueroa y Fierro, 1999). Donde $[\mathrm{T}]_{\mathrm{B}}^{\mathrm{B}}$ define la representación matricial de la transformación lineal $T$ en términos de la base $\mathrm{B}$ de $\mathrm{U}$.

Los corolarios son usados por matemáticos y algunos autores de libros de texto en el área, para presentar consecuencias directas de teoremas. Sin embargo algunos docentes en temáticas afines, pueden considerar los corolarios como casos particulares del teorema, que para otros docentes, matemáticos, autores de libros de texto, no es necesario destacar. Para el teorema que nos ocupa puede resultar importante resaltar la composición de una transformación lineal con ella misma $n$ veces, esto es $\left([\mathrm{T}]_{\mathrm{B}}^{\mathrm{B}}\right)^{\mathrm{n}}$ con $n \in \mathbb{N}$, cuestión que resulta imprescindible, por ejemplo en la teoría de los Sistemas Dinámicos.

\section{DESCRIPCIÓN DEL PENSAMIENTO MATEMÁTICO AVANZADO MODELADO POR LA TEORÍA APOE}

El centro de esta investigación se focaliza en el análisis cognitivo que modela la construcción (aprendizaje) del teorema TLMA, este análisis es denominado por la teoría APOE Descomposición Genética. A continuación se describen los principales constructos de este marco teórico, que fundamentan la investigación y que se usan para sustentar la interpretación del diseño hipotético del modelo cognitivo del teorema en estudio.

\section{La teoría APOE}

La teoría APOE es un marco teórico que busca describir e interpretar cognitivamente cómo los estudiantes construyen conceptos y/o nociones matemáticas. Sus fundamentos se basan en la Abstracción Reflexiva formulada por Piaget. Esta teoría ha sido usada y validada para explicar diferentes nociones y/o conceptos matemáticos avanzados, asociados con el nivel universitario; en áreas como cálculo (Valdivia y Parraguez, 2015; Trigueros y Martínez-Planell, 2010), álgebra lineal (Parraguez et al., 2016; Salgado y Trigueros, 2015; Parraguez y Oktaç, 2010), álgebra abstracta (Asiala et al., 1996) ecuaciones diferenciales (Trigueros, 2014), fractales (Villabona y Roa-Fuentes, 2016), probabilidad (Vásquez y Parraguez, 2014), teorema del isomorfismo (Mena et al., 2016), entre otros.

Esta teoría define las Acciones, Procesos, Objetos y Esquemas como las estructuras mentales que un estudiante puede lograr para construir un concepto y/o noción matemática; y los mecanismos de interiorización, coordinación, encapsulación y desencapsulación como medio para lograr dichas estructuras. En la figura 2, se representa en general cómo la comprensión de un concepto y/o noción matemática inicia cuando un estudiante aplica Acciones sobre Objetos previamente construidos (Arnon et al., 2014; Asiala et al., 1996). Estas relaciones son definidas por Dubinsky (1991) como un "circular feedback system". La complejidad de las relaciones que un estudiante puede lograr al desarrollar pensamiento matemático depende, desde la perspectiva de la teoría APOE, de las relaciones que logre establecer entre las diferentes estructuras mentales.

El aprendizaje de los estudiantes de un concepto, noción matemática o como en este caso de un teorema, inicia con la aplicación de Acciones sobre Objetos previamente construidos. Las Acciones son externas al estudiante dado que las realiza paso a paso y guiadas por instrucciones que no dependen de él. Para explicarlo se toma como ejemplo la transformación lineal (Roa-Fuentes y Oktaç, 2012); en este caso el estudiante requiere de una expresión que describa la función de forma analítica, para verificar el cumplimiento de la suma vectorial y el producto por un escalar mediante la transformación. Cuando dichas Acciones son repetidas con diferentes funciones y el estudiante puede reflexionar sobre ellas, pensando en las características de la función pero sin actuar de manera directa sobre ella, se dice que las Acciones han sido interiorizadas en un Proceso. Los Procesos son estructuras mentales sobre los cuales el estudiante tiene control. Los estudiantes que logran esta estructura pueden imaginar la aplicación de las Acciones sin necesidad de realizarlas paso a paso, incluso pueden saltar u obviar algunos. Por ejemplo, esta estructura de la transformación lineal, le permite al estudiante reflexionar sobre las características de funciones sencillas. Así de manera previa puede determinar si: muestra un contraejemplo en donde una de las dos propiedades de linealidad no se cumple o si demuestra la preservación de combinaciones lineales. 
Esquema

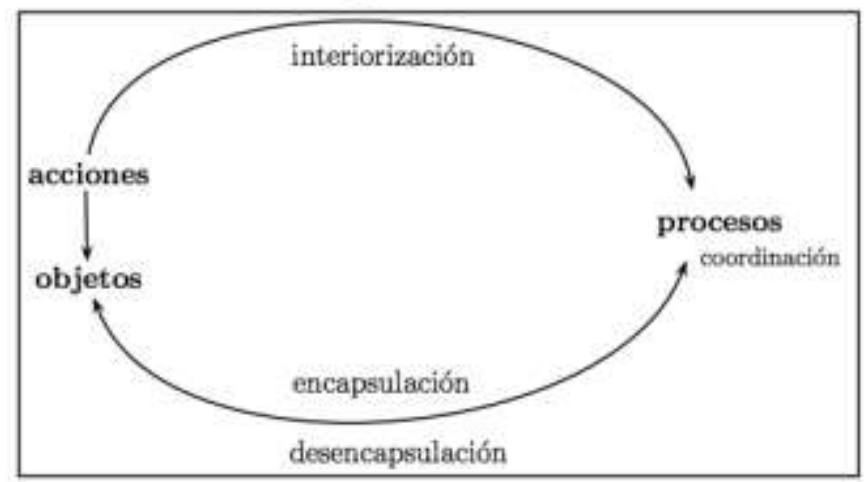

Fig. 2: Teoría APOE (Arnon et al., 2014)

La encapsulación de un proceso permite pasar de una estructura dinámica (Proceso) a una estática (Objeto). En el caso de la transformación lineal, la estructura Objeto en un estudiante puede evidenciarse cuando logra ver el conjunto de transformaciones lineales de un espacio en él mismo, como vectores de un espacio vectorial. $\mathrm{O}$ al generar nuevas transformaciones lineales gracias a las operaciones composición, suma o producto por un escalar, definidas para las funciones. Así el sistema definido en la figura 2 , inicia nuevamente y se da paso a la construcción de nuevos Objetos.

Cuando un estudiante aborda situaciones que propician la evolución de sus estructuras, es posible que se inicie la construcción del Esquema o que el Objeto construido sea asimilado por otro Esquema. Arnon et al., (2014) mencionan: "Específicamente, el Esquema de un individuo para cierto concepto matemático es la colección individual de Acciones, Procesos, Objetos y otros Esquemas, los cuales están vinculados por algunos principios generales o relaciones para formar un marco en la mente del individuo que puede ser aplicado en una situación problemática que implica ese concepto" (Arnon et al., 2014). En el caso de la transformación lineal, es primordial que el estudiante comprenda que las transformaciones lineales son básicamente funciones; esta es una característica fundamental de la estructura Objeto. Esto puede lograrse a partir del mecanismo de asimilación, que relaciona los Esquemas y los Objetos. Así un Objeto puede hacer parte de un Esquema previamente construido. Entonces gracias a la experiencia del estudiante con diferentes situaciones matemáticas que hacen referencia a esta relación, el Objeto transformación lineal es asimilado por el Esquema de función (Roa-Fuentes y Oktaç, 2010). Como consecuencia de esto último, los estudiantes comprenden que las transformaciones lineales son fundamentalmente funciones definidas entre espacios vectoriales que preservan combinaciones lineales.

Dubinsky y McDonald (2001), mencionan que la coherencia de un Esquema determina cuáles fenómenos están al alcance del estudiante y cuáles no. La experiencia de un estudiante con diferentes situaciones matemáticas asociadas a un concepto, genera la evolución de sus estructuras. Así un Esquema A puede ser tematizado en un Objeto (una estructura estática), de manera que pueda ser asimilado por un Esquema B. La asimilación de un Objeto (resultado de la encapsulación de un Proceso y la tematización de un Esquema) genera una reestructuración a partir del mecanismo de acomodación del Esquema $\mathrm{B}$, gracias a las nuevas relaciones que es posible establecer con el Objeto asimilado.

La tematización permite que todos los elementos que para un estudiante constituyen el Esquema de un fragmento específico de la matemática, se pongan juntos en una estructura estática. Por ejemplo, consideremos los Esquemas transformación lineal (Esquema A) y Función (Esquema B). El Esquema A puede ser tematizado de tal manera que todas las estructuras y mecanismos asociados con él se ponen juntos en un nuevo Objeto, de tal manera que los estudiantes consideran como funciones a las transformaciones lineales. Esquema B se acomoda y precisa como funciones aquellas relaciones entre espacios vectoriales que preservan combinaciones lineales. De esta manera los Esquemas evolucionan, mediante la interiorización de Acciones más sofisticadas y la encapsulación de Procesos enriquecidos; cada estructura inicial hace parte de estructuras cognitivas enriquecidas, que están en constante expansión por la experiencia del estudiante con nuevos problemas matemáticos. El grado de evolución de los Esquemas puede establecerse mediante la tríada Intra, Inter y Trans (Parraguez y Oktaç, 2012; Valdivia y Parraguez, 2014; Arnon et al., 2014; Parraguez, 2015). Un elemento importante en esta teoría para interpretar la construcción de un fragmento de conocimiento matemático, es la Descomposición Genética, que se define como un modelo hipotético que describe en detalle las estructuras y mecanismos mentales que son necesarios para que un estudiante aprenda un concepto matemático (Arnon et al., 2014). En el caso de esta investigación, interesa el diseño de una Descomposición Genética modelada a través de Esquemas cognitivos que describan la construcción y evolución del conocimiento incluido en el teorema TLMA. 


\section{DESCOMPOSICIÓN GENÉTICA DEL TEOREMA TLMA}

Las descomposiciones genéticas reportadas de los conceptos de espacio vectorial (Parraguez y Oktaç, 2012), base (Kú et al., 2008) y transformación lineal (Roa-Fuentes y Oktaç, 2012), son un insumo importante en la construcción de la descomposición genética el teorema TLMA. La estructura proceso de transformación lineal, descrita por Roa-Fuentes y Oktaç como una función que preserva combinaciones lineales es fundamental a la hora de construir el vector de coordenadas. La coordinación de dicho Proceso con el Proceso de base permite la construcción de la representación matricial de la transformación lineal tal como se describe más adelante.

Dado que la construcción de las estructuras mentales está descrita en términos de concepciones, conviene aclarar cierta diferencia. McDonald et al., (2000) definen la concepción como la idea personal que el estudiante tiene para comprender, a diferencia del concepto que se concibe como un acuerdo comunal establecido por la comunidad matemática. Iniciamos con el análisis de cuatro estructuras, que serán consideradas en el desarrollo de los niveles del Esquema del teorema TLMA.

1. Concepción Objeto de la composición de transformaciones lineales. Dadas dos funciones lineales $T: V \rightarrow$ $U$ y $L: U \rightarrow W$ el estudiante debe determinar si éstas pueden o no componerse. Carlson y Oehrtman (2015) muestran que "La composición es una coordinación de dos procesos de entrada y salida; el proceso de entrada por una función y el proceso de salida por una segunda función" (p.10). Resultado de esta coordinación se obtiene un nuevo proceso llamado $L \circ T: V \rightarrow W$. La encapsulación de este nuevo proceso en un Objeto puede generarse en un estudiante al preguntarle: ¿la función $L \circ T$ es lineal? Así puede construir esta nueva función como un elemento del espacio vectorial $L(V, W)$ definido como el conjunto de transformaciones lineales de $V$ en $W$.

2. Concepción Proceso de vector de coordenadas. En la construcción del concepto vector de coordenadas entran en juego una concepción proceso de espacio vectorial (Parraguez y Oktaç, 2012) y de base ordenada (Kú et al., 2008). Supongamos una base $\beta=\left\{u_{1}, u_{2}, \ldots, u_{n}\right\}$ del espacio vectorial $U$, y una concepción proceso de base que le permite al estudiante construir un vector $u$ en $U$ como combinación lineal de los vectores de $\beta$, tal que $u=c_{1} u_{1}+c_{2} u_{2}+\cdots+c_{n} u_{n}$. El vector $u$ se construye como un proceso en términos de los vectores de la base $\beta$, en donde los escalares $c_{1}, c_{2}, \ldots c_{n}$ lo definen de manera única. Si el estudiante desarrolla diferentes situaciones en donde varía la base, al encontrar los escalares y escribir la combinación lineal puede interiorizar estas acciones en el proceso vector de coordenadas. Una concepción proceso del vector de coordenadas, permite que el estudiante construya el vector $u$ como un elemento dependiente de los vectores de la base, que a su vez determinan los escalares de la combinación; de tal manera que el vector $u$ ahora es visto como el vector de coordenadas $[u]_{\beta}$ definido como lo muestra la ec. 1:

$[u]_{\beta}=\left[\begin{array}{c}c_{1} \\ c_{2} \\ \vdots \\ c_{n}\end{array}\right]$

La encapsulación de este proceso puede generarse cuando el estudiante trabaja sobre espacios vectoriales diferentes a $\mathbb{R}^{n}$. La concepción Objeto, se caracteriza por la construcción del isomorfismo entre cualquier espacio vectorial de dimensión $n$ y $\mathbb{R}^{n}$; así el vector $[u]_{\beta}$ es visto como un todo, como un vector del espacio $\mathbb{R}^{n}$. Sobre este nuevo Objeto es posible aplicar nuevas acciones, por ejemplo al construir la matriz de cambio de base.

3. Esquema de transformación lineal. Esta estructura está conformada principalmente por estructuras relacionadas con la transformación lineal, como una función que preserva combinaciones lineales (RoaFuentes y Oktaç, 2012). Así dadas las imágenes bajo la transformación lineal, digamos $T$ de los vectores de una base de $U$, la transformación queda definida para todos los vectores de $U$. La construcción de la matriz que representa dicha transformación lineal, está determinada precisamente por la fijación de las bases de los espacios sobre los cuales se define $T$.

4. Concepción Objeto de la Representación Matricial de una transformación lineal. Cuando un estudiante logra una concepción Objeto del vector de coordenadas puede construir la matriz que representa una transformación lineal. Una concepción Acción de la representación matricial le permite al estudiante calcular la matriz en términos de las bases canónicas de los espacios; construyendo cada columna de la matriz como el vector de coordenadas correspondiente. Esto es (siguiendo con la notación de la figura 3): selecciona una base $\beta_{1}=\left\{u_{1}, u_{2}, \ldots, u_{n}\right\}$ del espacio vectorial $U$ y una base $\beta_{2}=\left\{v_{1}, v_{2}, \ldots, v_{m}\right\}$ del espacio vectorial $V$, 
entonces puede construir la matriz $A=\left[\left[\mathrm{T}\left(u_{1}\right)\right]_{\beta_{2}}\left[\mathrm{~T}\left(u_{2}\right)\right]_{\beta_{2}} \ldots\left[\mathrm{T}\left(u_{n}\right)\right]_{\beta_{2}}\right] \in M_{m \times n}$. A partir de estas Acciones el estudiante reflexiona sobre la importancia de la base, por ejemplo, al calcular diferentes matrices para la misma función variando bases ordenas de los espacios involucrados. Esto refleja una concepción Proceso de la representación matricial. La encapsulación de este Proceso está determinada por la aplicación de nuevas transformaciones sobre la matriz; por ejemplo cuando el estudiante puede calcular la imagen de un vector $v$, mediante la operación $A v=T(v)$. La representación matricial de una transformación lineal, puede construirse a partir de la asimilación de una matriz $A_{m \times n}$ como un Objeto por el Esquema de transformación lineal (ver Figura 3). La asimilación se genera gracias a la elección que el estudiante hace de una base ordenada de $U$ o se genera a partir de la importancia que el estudiante asigne a las bases de los espacios $U$ y $V$ planteados en una situación matemática. El tamaño de la matriz $A$ queda definido por la dimensión de los espacios $U$ y $V$. Así el Esquema de transformación lineal se acomoda y el estudiante comprende que una transformación lineal puede ser una función que preserva combinaciones lineales o una matriz.

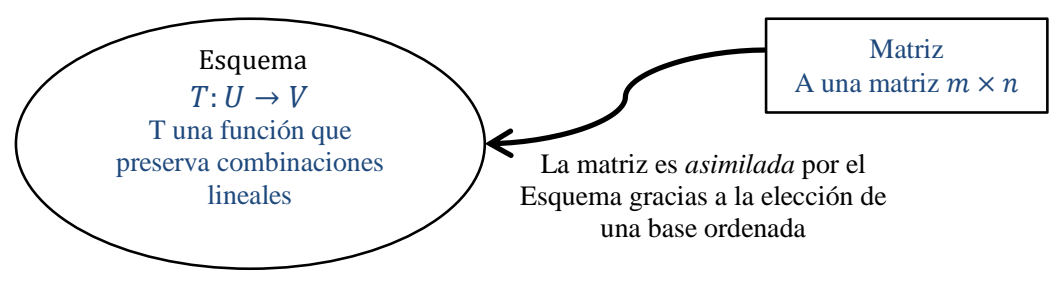

Fig. 3: La matriz A es asimilada por el Esquema de transformación lineal

Con estas cuatro estructuras se da paso a la construcción del Esquema del teorema TLMA. Para esto se toma en cuenta la definición general de los niveles Intra, Inter y Trans (Arnon et al., 2014), además de los indicadores de desarrollo definidos por Parraguez (2015) para el Esquema de los conceptos básicos de álgebra lineal. "Desde estas caracterizaciones generales, un indicador del desarrollo del Esquema en la mente de un estudiante es el tipo de relaciones (Intra), el tipo de transformaciones (Inter) y el tipo de conservaciones o invariantes (Trans) que los estudiantes son capaces de establecer entre los estados de construcción de conceptos del álgebra lineal particulares, que evocan dentro del Esquema, cuando resuelven problemas" (Parraguez, 2015). Cada tipo de indicador puede evidenciar la manera como un estudiante evoca su Esquema del teorema TLMA.

En particular lo que refiere a la construcción de teoremas es pertinente resaltar su uso, no como la aplicación de un "slogan" (Abramovitz et al., 2009) sino como lo propone Parraguez (2014), esto es, como "un medio de construcción conceptual". Un estudiante al abordar un problema en álgebra lineal puede determinar si usa o no el teorema TLMA. Cuando tiene las estructuras previas, es posible que ese uso permita la construcción conceptual del teorema TLMA y se de paso a la evolución de su Esquema. En la Tabla 1 se muestra un análisis inicial que caracteriza dicha evolución.

\section{MÉTODO}

Esta investigación tiene por objetivo determinar las construcciones y mecanismos mentales que subyacen a la construcción del teorema TLMA, en estudiantes universitarios. La forma de indagación que permite determinar las construcciones, requirió de registros de observación de las producciones escritas de los estudiantes, y del análisis de sus procedimientos. Para examinar dichos procedimientos, consideramos que una aproximación adecuada es el Estudio de Casos (Stake, 2010), ya que es particularmente apropiada para examinar una situación en profundidad y en un período de tiempo acotado. Los estudios de casos que se consideran en la investigación se insertaron dentro del ciclo de investigación de la teoría APOE (Arnon et al., 2014), para situar las características de los estudiantes participantes.

\section{Participantes}

Las unidades de estudio fueron 6 alumnos chilenos de una universidad del centro del país, y 6 estudiantes colombianos de un estado del centro del país, que son considerados como dos casos (Stake, 2010). Estos casos (ver Tabla 2) se justifican por la necesidad de delimitar aquellas nuevas etapas y categorías, que permiten focalizar la investigación con mayor precisión, hacia las concepciones matemáticas presentes en el conocimiento del teorema TLMA. Además esta perspectiva metodológica sustenta el ejercicio investigativo propuesto, que busca describir lo más explícitamente posible las estructuras y los mecanismos mentales, que mostraron los estudiantes dispuestos en los casos de estudio, particularmente en situaciones matemáticas que se relacionan con el teorema TLMA. Esto se sustenta desde la heterogeneidad de la formación de los estudiantes en distintas universidades de Sudamérica. 
Tabla 1: Niveles de evolución del Esquema del teorema TLMA

\begin{tabular}{|c|c|c|}
\hline Nivel & Características Generales (Arnon et al., 2014) & Teorema TLMA y su Corolario \\
\hline $\begin{array}{l}\text { Intra- } \\
\text { TLMA }\end{array}$ & $\begin{array}{l}\text { El desarrollo Intra del Esquema se caracteriza } \\
\text { por un enfoque en Acciones, Procesos y } \\
\text { Objetos individuales aislados de otros } \\
\text { elementos cognitivos de naturaleza similar. }\end{array}$ & $\begin{array}{l}\text { En el nivel Intra-TLMA, los estudiantes empiezan a } \\
\text { identificar diferentes relaciones lógicas en términos de } \\
\text { cuantificadores y/o existenciales entre las estructuras } \\
\text { previas que pueden ser o no correctas. Esto depende de la } \\
\text { construcción de la función composición de transformaciones } \\
\text { lineales como una nueva transformación lineal y la relación } \\
\text { entre sus representaciones matriciales a partir del producto. } \\
\text { En este nivel sólo se consideran las relaciones entre } \\
\text { espacios definidos a través de las bases canónicas de } \mathbb{R}^{n} \text {. } \\
\text { El nivel Intra del corolario se describe a través de la base } \\
\text { canónica cuando se presenta la necesidad de que } L \text { sea } \\
\text { igual a } T \text { en el teorema. }\end{array}$ \\
\hline $\begin{array}{l}\text { Inter- } \\
\text { TLMA }\end{array}$ & $\begin{array}{l}\text { El nivel Inter se caracteriza por la construcción } \\
\text { de relaciones y transformaciones entre las } \\
\text { estructuras cognitivas que componen el } \\
\text { Esquema, donde un individuo puede } \\
\text { comenzar a agrupar elementos e incluso } \\
\text { llamarlos con el mismo nombre. }\end{array}$ & $\begin{array}{l}\text { En el nivel Inter-TLMA los estudiantes pueden realizar } \\
\text { transformaciones sobre las transformaciones lineales como } \\
\text { funciones y/o su representación matricial gracias a las } \\
\text { relaciones establecidas en el nivel anterior. Dichas } \\
\text { transformaciones están determinadas por la fijación de } \\
\text { bases ordenadas diferentes a la canónica. Esto le permite al } \\
\text { estudiante construir nuevas funciones lineales a partir del } \\
\text { producto de sus representaciones matriciales y viceversa a } \\
\text { partir de la composición de funciones. La relación construida } \\
\text { entre la transformación lineal y su representación como } \\
\text { matriz es general, lo que permite realizar transformaciones } \\
\text { sobre operaciones. } \\
\text { El nivel Inter del corolario se describe a través de la base } \\
\text { que se ha asignado cuando se presenta la necesidad de } \\
\text { que } L \text { sea igual a } T \text { en el teorema. }\end{array}$ \\
\hline $\begin{array}{l}\text { Trans- } \\
\text { TLMA }\end{array}$ & $\begin{array}{l}\text { En el nivel Trans el individuo construye una } \\
\text { estructura subyacente implícita o explícita a } \\
\text { través de la cual se entienden las relaciones } \\
\text { desarrolladas en el nivel Inter y por la cual el } \\
\text { Esquema alcanza la coherencia que se } \\
\text { determina por la capacidad del individuo para } \\
\text { decidir lo que está al alcance del Esquema y } \\
\text { lo que no. }\end{array}$ & $\begin{array}{l}\text { En el nivel Trans-TLMA el estudiante puede determinar los } \\
\text { constructos que son invariantes y definir el teorema TLMA. } \\
\text { La coherencia del Esquema le permite determinar la } \\
\text { conservación de la representación matricial de } L \text {. } T \text { una vez } \\
\text { fijadas las bases. Además puede admitir las condiciones en } \\
\text { que éste puede ser aplicado en la solución de una situación. } \\
\text { Los invariantes están definidos para situaciones que } \\
\text { involucran espacios vectoriales de dimensión finita y } \\
\text { funciones lineales. Este uso de la forma general del teorema } \\
\text { induce su demostración, en donde se ratifican las relaciones } \\
\text { invariantes. } \\
\text { El nivel Trans del corolario se describe a través de su } \\
\text { aplicación para } n \text { potencias de la función composición a } \\
\text { través del producto } n \text {-veces de la matriz. }\end{array}$ \\
\hline
\end{tabular}

Específicamente los casos lo constituyen estudiantes de las carreras de Pedagogía y Licenciatura en Matemáticas. El procedimiento seguido para la selección de los estudiantes, utilizó las siguientes categorías: Heterogeneidad en la formación de los estudiantes que pertenecen al conjunto; Existencia de programas de Pedagogía en Matemática y de Licenciatura en Matemática, que incluyan al menos una asignatura de álgebra lineal; Accesibilidad de los investigadores; Avance curricular: que los estudiantes no hayan reprobado las asignaturas de álgebra básica y cursen a lo menos el sexto semestre de su primera carrera; para descartar en lo posible que sus construcciones mentales se limiten a acciones y/o procesos. Los 12 estudiantes fueron etiquetados por EC\# para el primer caso y ECO\# para el segundo, respectivamente; la Tabla 2, resume la información por caso.

Los casos de estudio se eligieron entre los estudiantes con quienes se aplicó el ciclo de investigación previsto en la teoría APOE. Este ciclo establece el desarrollo de tres componentes: un Análisis Teórico, que da lugar al planteamiento de una Descomposición Genética; Diseño e implementación de un modelo de enseñanza, basado en la Descomposición Genética, y la tercera componente Recolección, Observación y Análisis de datos, que se presenta en este escrito mediante el estudio de casos descrito. La verificación se llevó a cabo por los investigadores, de manera independiente en primer término y mediante la negociación del análisis para triangular 
los resultados (Arnon et al., 2014). Los estudiantes de los dos casos de estudio habían cursado una materia de álgebra lineal que incluye básicamente los conceptos de espacio vectorial, combinación lineal, base, dependencia e independencia lineal, transformaciones lineales $y$, valores y vectores propios. El desarrollo del curso estuvo guiado por profesores con amplia experiencia en un contexto de enseñanza tradicional, en donde el profesor ofrece explicaciones en el tablero y resuelve problemas asociados con el contenido visto.

Tabla 2: Resumen de la recolección de información por casos de estudio

\begin{tabular}{|l|l|l}
\hline Tipo de estudiante & \multicolumn{1}{|c|}{$\begin{array}{c}\text { Caso 1 } \\
\text { Estudiantes de la Universidad chilena } \\
\text { EC1, EC2, .., EC6 }\end{array}$} & $\begin{array}{l}\text { Caso 2 } \\
\text { Estudiantes de la Universidad colombiana } \\
\text { ECO1, ECO2, ..., ECO6 }\end{array}$ \\
\hline
\end{tabular}

La aplicación de este ciclo permitió obtener una descripción de las estructuras y mecanismos mentales que mostraron los estudiantes, dispuestos en casos de estudio. La Descomposición Genética validada, a través de la descripción de cada nivel del Esquema del teorema TLMA, es el resultado de la aplicación completa de las tres componentes de este ciclo, que permitió documentarla con datos empíricos, y en algunos aspectos específicos refinarla, para que diera cuenta de la construcción real del teorema TLMA.

\section{ANÁLISIS DEL CUESTIONARIO}

Se diseñó un cuestionario de 5 preguntas (Tabla 3), con la intención de documentar las estructuras y mecanismos mentales planteados en el análisis teórico preliminar, interpretado a través de los niveles del Esquema del teorema TLMA y su corolario como un caso particular de este último. El cuestionario fue desarrollado por los estudiantes un semestre después de haber terminado su primer curso de álgebra lineal. Se realizó un análisis a priori y otro a posteriori para cada una de las preguntas del cuestionario. Los dos fueron profusamente discutidos entre las investigadoras y las discrepancias se negociaron hasta alcanzar un acuerdo.

Tabla 3: Preguntas del cuestionario

\begin{tabular}{|c|c|}
\hline Pregunta 1 & $\begin{array}{l}\text { Sean } f \text { y } g \text { transformaciones lineales definidas de } \mathbb{R}^{2} \text { en } \mathbb{R}^{2} \text { tales que: } \\
\qquad f(x, y)=(2 x+y, x-y) \\
\qquad g(x, y)=(x+y, x) \\
\text { a. Encuentra la representación matricial de } f \text { y la representación matricial de } g . \\
\text { b. Calcula la representación matricial de } f \circ g \text { y } g \circ f .\end{array}$ \\
\hline Pregunta 2 & $\begin{array}{l}\text { Sea } g: M_{2}(\mathbb{R}) \rightarrow \mathbb{R}^{5} \text { una transformación lineal, donde } B=\left\{e_{1}, e_{2}, e_{3}, e_{4}\right\} \text { y } B^{\prime}=\left\{e_{1}^{\prime}, e_{2}^{\prime}, e^{\prime}, e^{\prime}{ }_{4}, e^{\prime}{ }_{5}\right\} \text { son las } \\
\text { bases canónicas de } M_{2}(\mathbb{R}) \text { y } \mathbb{R}^{5} \text { respectivamente tal que } g\left(e_{1}\right)=(2,0,0,0,2), g\left(e_{2}\right)=(0,1,0,0,0), g\left(e_{3}\right)= \\
(0,-3,0,1,0) \text { y } g\left(e_{4}\right)=(0,0,0,0,0) \text {. } \\
\text { a. Determina } g\left(\begin{array}{cc}x & y \\
z & w\end{array}\right) \text { para todo }\left(\begin{array}{cc}x & y \\
z & w\end{array}\right) \text { en } M_{2}(\mathbb{R}) . \\
\text { b. Determina }[g]_{B}^{B \prime} \text { y }\left[\left(\begin{array}{cc}x & y \\
z & w\end{array}\right)\right]_{B} \\
\text { c. Calcula } g\left(\begin{array}{cc}1 & -1 \\
0 & 3\end{array}\right)\end{array}$ \\
\hline Pregunta 3 & $\begin{array}{l}\text { Sea } T: V \rightarrow V \text { una transformación lineal y } B \text { una base de } V \text {. Si }[T]_{B}^{B}=\left(\begin{array}{ll}0 & 1 \\
2 & 3\end{array}\right) \text {, entonces } i[T \circ T]= \\
\left(\begin{array}{ll}0 & 1 \\
4 & 9\end{array}\right) \text { ? Justifica ampliamente tu respuesta. }\end{array}$ \\
\hline Pregunta 4 & $\begin{array}{l}\text { Escribe dos transformaciones lineales } f \text { y } g \text { tal que las funciones } f \circ g \text { y } g \circ f \text { estén definidas. } \\
\text { a. Encuentra las funciones } f \circ g \text { y } g \circ f \text {. } \\
\text { b. Calcula la representación matricial de las funciones } f, g, f \circ g \text { y } g \circ f \text {. }\end{array}$ \\
\hline Pregunta 5 & $\begin{array}{l}\text { Define los conceptos Transformación Lineal y Representación Matricial de una Transformación Lineal. } \\
\text { ¿Conoces algún resultado que relacione dichos conceptos? Si tu respuesta es positiva explica cuál. } \\
\text { Puedes plantear ejemplos específicos o presentar generalidades sobre tus argumentos. }\end{array}$ \\
\hline
\end{tabular}

\section{Análisis a priori de las preguntas del cuestionario}

El análisis a priori incluye una descripción para las 5 preguntas del cuestionario, tomando como referente las tres estructuras previas descritas en la Descomposición Genética, así como los niveles del Esquema que los estudiantes pueden modelar para comprender el teorema TLMA. 
La Pregunta 1 del cuestionario requiere que el estudiante proponga explícitamente una base $\beta$ para $\mathbb{R}^{2}$, podría ser la canónica $C$ para aplicar acciones sobre el Objeto definido $C$ a través de $f$ y $g$, obteniendo como resultado el Objeto de la representación matricial en $C,[f]_{c}=\left(\begin{array}{cc}2 & 1 \\ 1 & -1\end{array}\right)$ y $[\mathrm{g}]_{\mathrm{c}}=\left(\begin{array}{cc}1 & 1 \\ 1 & -0\end{array}\right)$. Paso seguido, los estudiantes pueden establecer la relación entre la composición de transformaciones lineales con el producto de su representación matricial $[\mathrm{f} \circ \mathrm{g}]_{\beta}=[\mathrm{f}]_{\beta} \cdot[\mathrm{g}]_{\beta}$. En este caso resulta natural que los estudiantes elijan la base canónica, lo que es fundamental es que se evidencie la elección de dicha base. Los procedimientos de los estudiantes pueden evidenciar un nivel Intra del teorema TLMA.

La Pregunta 2 define la transformación lineal g a través de las imágenes de los vectores de la base canónica $\mathrm{B}$ de $\mathrm{M}_{2}(\mathbb{R})$, lo que sitúa el trabajo del estudiante en el Esquema Intra-TLMA. Con esta información debe determinar la forma analítica y matricial de g, a través de reconocer relaciones entre el Objeto de la representación matricial de la transformación lineal $g$; esto es, $[\mathrm{g}(\mathrm{x}, \mathrm{y})]_{\mathrm{B}}^{\mathrm{B}^{\prime}}=[\mathrm{g}]_{\mathrm{B}}^{\mathrm{B}}[(\mathrm{x}, \mathrm{y})]_{\mathrm{B}}$ donde $[\mathrm{g}]_{\mathrm{B}}^{\mathrm{B}^{\prime}}=\left(\left[\mathrm{g}\left(\mathrm{e}_{1}\right)\right]_{\mathrm{B}^{\prime}}\left[\mathrm{g}\left(\mathrm{e}_{2}\right)\right]_{\mathrm{B}^{\prime}}\left[\mathrm{g}\left(\mathrm{e}_{3}\right)\right]_{\mathrm{B}^{\prime}}\left[\mathrm{g}\left(\mathrm{e}_{4}\right)\right]_{\mathrm{B}^{\prime}}\right)$. Las acciones específicas que un estudiante pueda realizar para determinar la transformación lineal, pueden evidenciar la coordinación del proceso de base con la transformación lineal (función o matriz). Este mecanismo es fundamental para lograr la construcción del teorema TLMA en su nivel Intra. Si la función (transformación lineal) ha sido construida como una función que "preserva combinaciones lineales" la matriz (cuyo tamaño está determinado por la dimensión de los espacios dominio y recorrido) será asimilada por el Esquema de transformación lineal. De tal manera que dado un vector del conjunto de salida de la transformación lineal, un estudiante podrá determinar su imagen a través de aplicar la función al vector o multiplicando la matriz por dicho vector. La hipótesis que se propone es, si un estudiante no ha construido el concepto coordenada de la imagen de un vector como Objeto, tendrá dificultades para realizar las acciones necesarias sobre este para construir la matriz asociada a la transformación lineal.

La clara relación que existe entre la forma analítica y matricial de la transformación lineal hace que en algunas situaciones sea inseparable su análisis. La manera como cada estudiante abordará cada pregunta, parece estar predeterminada por la forma en que el concepto aparece en ella. En el caso de las preguntas propuestas, ver tabla 3 , se puede distinguir que las preguntas 2 y 3 hacen mayor énfasis en la transformación lineal como una matriz.

En la Pregunta 3 el estudiante requiere evocar el corolario del teorema TLMA, con apoyo en una concepción Objeto de la transformación lineal como función, además de una concepción Objeto de la representación matricial que le permita determinar $[\mathrm{T} \circ \mathrm{T}]$ a partir del producto matricial. La relación entre componer funciones y multiplicar matrices es propia de un nivel Intra-TLMA, si la base considerada es la canónica e Inter-TLMA si no lo es.

Para la Pregunta 4 los estudiantes deben pensar en dos funciones lineales $f$ y $g$; en primer lugar teniendo en cuenta las condiciones que deben cumplir sobre el dominio y recorrido para que las operaciones $f \circ g$ y $g \circ f$ queden definidas. Evocando bases para los espacios vectoriales sobre los que se define $f$ y $g$, esto puede relacionarse con la representación matricial de las transformaciones lineales. En este caso los estudiantes podrían determinar el tamaño de las matrices de tal manera que el producto esté definido. Este tipo de pensamiento se asocia con la forma dinámica de una concepción proceso de la representación matricial para $f$ y $g$. Sin embargo, cuando se formulan las funciones y se generan nuevos Objetos, podemos encontrar evidencia de una concepción Objeto de la representación matricial para $f$ y $g$; gracias a la construcción de un nuevo Objeto en su forma estática. Esto permite evidenciar el nivel Intra del Esquema del teorema TLMA, si la bases explicitadas en el argumento son las canónicas, o nivel Inter del Esquema TLMA en otro caso.

La intención de la Pregunta 5 en el instrumento, es mostrar los elementos que los estudiantes están evocando de los niveles Intra-TLMA, Inter-TLMA o Trans-TLMA para construir relaciones, transformaciones o invariantes de tópicos matemáticos, que más adelante como resultado de esta investigación se etiquetan como indicadores de cada uno de los niveles del Esquema del teorema TLMA.

\section{RESULTADOS}

Los resultados se enmarcan en el análisis del trabajo evidenciado por los estudiantes al responder el cuestionario de manera escrita durante un tiempo aproximado de 120 minutos. Dicho análisis toma como referente el tipo de estructuras mentales que los estudiantes evidenciaron en los procedimientos realizados, a través de los argumentos observables que se interpretan con base en sus respuestas y reflexiones con los investigadores sobre sus estrategias. 
Concepción Objeto de la Representación Matricial de una Transformación Lineal: Asimilación del Objeto matriz por el Esquema de Transformación Lineal. En la Descomposición Genética describimos cuatro estructuras previas necesarias para la construcción del Esquema del teorema TLMA; estas son: una concepción Objeto de composición de transformaciones lineales, una concepción Objeto de vector de coordenadas, una concepción Objeto de la representación matricial de una transformación lineal y un Esquema de transformación lineal. El análisis de los datos evidencia que estas estructuras se relacionan por medio de la fijación de una base ordenada, que define una representación matricial de la transformación lineal en su forma funcional.

Todos los estudiantes del Caso 1 mostraron una concepción Objeto de la representación matricial de la transformación lineal. Por ejemplo para la pregunta 1, EC1 fija las bases canónicas para determinar las imágenes de sus vectores; su procedimiento se describe en la Tabla 4.

Ahora, el hecho de construir la matriz dada la transformación lineal en su forma analítica, puede considerarse como una forma dinámica de la matriz, que responde a construir los vectores de coordenadas, que dependen de las bases elegidas. Pero la importancia de la construcción de esta matriz como un Objeto, va más allá de su cálculo. Para esto, en la Tabla 5 se muestra el trabajo realizado por ECO2 sobre la pregunta 2 .

Tabla 4: Procedimientos de EC1 en la pregunta 1

\begin{tabular}{|c|c|}
\hline $\begin{array}{l}\text { Problema } 1 \\
\text { EC1 calcula la imagen de los vectores de la }\end{array}$ & $\begin{array}{l}\text { i) } A=\{(1,0),(0,1)\}, \text { base de } \mathbb{R}^{2} \\
f(1,0)=(2,1) \quad f(0,1)=(1,-1) \\
{[f]_{A}^{A}=\left[\begin{array}{cc}2 & 1 \\
1 & -1\end{array}\right]}\end{array}$ \\
\hline$[f]_{A}^{A}$ у $[g]_{A}^{A}$ & $\begin{array}{l}\text { ii) } g(1,0)=(1,1) \quad g(0,1)=(1,0) \\
{[g]_{A}^{A}=\left[\begin{array}{ll}1 & 1 \\
1 & 0\end{array}\right]}\end{array}$ \\
\hline
\end{tabular}

Tabla 5: Procedimientos de ECO2 en la pregunta 2

\begin{tabular}{|c|c|}
\hline Problema 2 & 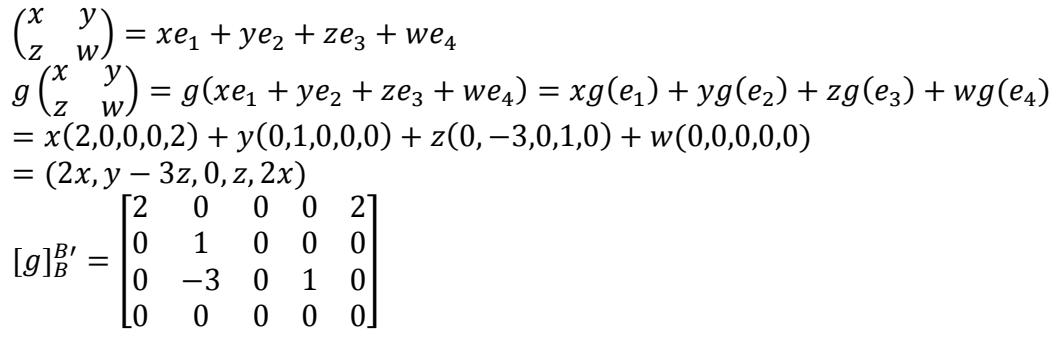 \\
\hline
\end{tabular}

En el análisis mostrado por ECO2 resulta fundamental el andamiaje de las estructuras mentales previas para el aprendizaje del teorema TLMA como un Esquema. En general, la reflexión de los estudiantes de ambos casos, propicia la relación entre las características de la forma analítica de la transformación lineal con su forma matricial. Específicamente en el trabajo de ECO2 se evidencia la preservación de combinaciones lineales de $g$, sin embargo, no evoca explícitamente indicios de relaciones entre las dimensiones de los espacios $M_{2}(\mathbb{R})$ y $\mathbb{R}^{5}$ que forman parte de los datos de la pregunta. Por tanto no calcula la matriz que representa la función $g$ sino su transpuesta. Esto es una evidencia de que no ha logrado una concepción Proceso de la representación matricial de la transformación lineal. Dado que no relaciona el tamaño de la matriz con las dimensiones de los espacios involucrados.

Una característica común de los estudiantes del Caso 2 es que sus procedimientos son mecánicos, no hacen alusión al uso de teoremas o resultados generales asociados con los elementos descritos inicialmente en la Descomposición Genética, que dan paso a la construcción del Esquema del teorema TLMA. El análisis de la pregunta 5, muestra que sus concepciones aún son primarias y por tanto no pueden escribir resultados que relacionen las representaciones matriciales y analíticas de una transformación lineal. Incluso sus definiciones de la transformación lineal en su forma analítica y matricial no se acercan a las formales. Por ejemplo ECO5 escribe: "Transformación lineal es una función que debe estar bien definida. Representación matricial. Sea $\left(x_{1}, x_{2}, \ldots, x_{n}\right) \in \mathbb{R}^{n}$ la representación matricial consiste en ubicar componente a componente los elementos de un vector en una matriz". A continuación se muestran evidencias de la construcción de los niveles del Esquema TLMA (Intra, Inter, Trans) a través de la descripción de sus indicadores (Relaciones, Transformaciones e Invariantes, respectivamente). 
Evidencias de la construcción del Esquema del teorema en un nivel Intra-TLMA. Para el Caso 1 dos de los estudiantes (EC1, EC6) aplicaron el teorema TLMA; en la Tabla 6 se puede ver el procedimiento realizado por el estudiante EC6 al responder la parte b de la pregunta 1.

Tabla 6: Procedimientos de EC6 en la pregunta 1.b

\begin{tabular}{|c|c|}
\hline $\begin{array}{c}\text { Pregunta } 1 \\
\text { Relaciones entre } \\
\text { composición de TL y MA }\end{array}$ & 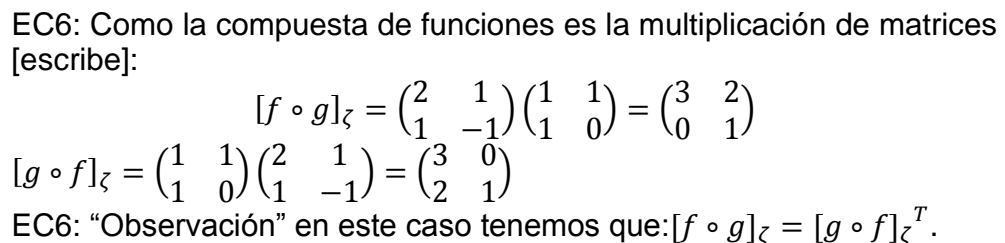 \\
\hline
\end{tabular}

Antes de iniciar la solución de este problema, el estudiante EC6 define el conjunto $\zeta$ como la base canónica de $\mathbb{R}^{2}$. Esta es una característica común en los estudiantes del Caso 1; además EC6 identifica una relación entre las matrices resultantes. Como mostramos en el análisis a priori del cuestionario, involucrar el concepto de base es fundamental en la construcción de la matriz que representa la transformación lineal, pues sitúa al estudiante en el primer nivel del Esquema del teorema TLMA. Las relaciones en este nivel están determinadas por la asociación que se logra establecer entre la representación analítica y matricial de la transformación lineal y el vehículo para lograr esa correspondencia es la base.

Evidencias de la construcción del Esquema del teorema en un nivel Inter-TLMA. Los estudiantes del Caso 1 logran abordar la pregunta 2 sin dificultad, sólo los estudiantes EC1 y EC2 cometen errores al reemplazar o calcular dentro del procedimiento. Se destaca el trabajo del estudiante EC3, quien resuelve la pregunta haciendo énfasis sobre la transformación lineal como función y como matriz (ver Tabla 7).

Tabla 7: Procedimientos de EC3 en la pregunta 2

\begin{tabular}{|l|l|}
\hline & EC3: Por Teorema fundamental de las transformaciones existe una única $g \in$ \\
& $L\left(M_{2}(\mathbb{R}), \mathbb{R}^{5}\right)$ tal que: \\
& $\operatorname{Sea}\left(\begin{array}{cc}x & y \\
z & w\end{array}\right) \in \mathcal{M}_{2}(\mathbb{R}):\left(\begin{array}{cc}x & y \\
z & w\end{array}\right)=x\left(\begin{array}{ll}1 & 0 \\
0 & 0\end{array}\right)+y\left(\begin{array}{ll}0 & 1 \\
0 & 0\end{array}\right)+z\left(\begin{array}{ll}0 & 0 \\
1 & 0\end{array}\right)+w\left(\begin{array}{ll}0 & 0 \\
0 & 1\end{array}\right)$ \\
$\Rightarrow$ & $g\left(\begin{array}{cc}x & y \\
z & w\end{array}\right)=x g\left(\begin{array}{ll}1 & 0 \\
0 & 0\end{array}\right)+y g\left(\begin{array}{ll}0 & 1 \\
0 & 0\end{array}\right)+z g\left(\begin{array}{ll}0 & 0 \\
1 & 0\end{array}\right)+w g\left(\begin{array}{ll}0 & 0 \\
0 & 1\end{array}\right)$ \\
& $\Rightarrow g\left(\begin{array}{ll}x & y \\
z & w\end{array}\right)=x(2,0,0,0,2)+y(0,1,0,0,0)+z(0,-3,0,1,0)+w g(0,0,0,0,0)$ \\
& $\Rightarrow g\left(\begin{array}{ll}x & y \\
z & w\end{array}\right)=(2 x, y-3 z, 0, z, 2 x)$.
\end{tabular}

Los procedimientos de EC3 muestran que tiene completo control sobre las Acciones que aplica sobre los Objetos dados. Incluso para calcular la matriz, no lo hace de manera mecánica sino que escribe los vectores imagen como columnas, de tal manera que puede definir la matriz. En la Tabla 8 aparece el procedimiento realizado por este estudiante.

Tabla 8: Evidencias de construcción del nivel Inter-TLMA

\begin{tabular}{|l|c|}
\hline \multicolumn{1}{|c|}{ Pregunta 2} & {$[g]_{B}^{B^{\prime}}=\left(\left[g\left(e_{1}\right)\right]_{B^{\prime}}\left[g\left(e_{2}\right)\right]_{B^{\prime}}\left[g\left(e_{3}\right)\right]_{B^{\prime}}\left[g\left(e_{4}\right)\right]_{B^{\prime}}\right)$} \\
Transformación de las coordenadas de & $=\left(\begin{array}{cccc}2 & 0 & 0 & 0 \\
0 & 1 & -3 & 0 \\
0 & 0 & 0 & 0 \\
0 & 0 & 1 & 0 \\
2 & 0 & 0 & 0\end{array}\right)$ \\
\hline las imágenes de acuerdo a las bases. & \\
\hline
\end{tabular}

De sus escritos se puede interpretar que EC3 cuenta con las relaciones previas suficientes para transformar las coordenadas de las imágenes de acuerdo a las bases, lo que sitúa su trabajo en la construcción del Esquema del teorema TLMA en un nivel Inter.

Evidencias del Nivel Inter del Corolario: La pregunta 3, hace referencia de manera específica al Corolario del teorema TLMA situando al estudiante en un nivel Inter de trabajo, por las características de su enunciado, esto es, $-B$ una base de $V$-. 
Los estudiantes del Caso 1, muestran evidencias de esta estructura, dado que de manera explícita hacen alusión al uso del teorema TLMA en su caso particular. Por ejemplo ver el procedimiento de EC3 que aparece en la Tabla 9.

Tabla 9: Evidencias del Nivel Inter del Corolario

\section{Pregunta 3}

EC3 relaciona el teorema TLMA con un caso particular, cuando $L=T$, que corresponde al enunciado del corolario.
EC3: Por el teorema de matriz de composición de transformaciones lineales:

$$
\begin{gathered}
{[T \circ T]_{B}^{B}=[T]_{B}^{B} \cdot[T]_{B}^{B}} \\
=\left(\begin{array}{cc}
0 & 1 \\
2 & 3
\end{array}\right)\left(\begin{array}{cc}
0 & 1 \\
2 & 3
\end{array}\right) \\
=\left(\begin{array}{cc}
2 & 3 \\
6 & 11
\end{array}\right)
\end{gathered}
$$

Para el nivel Inter del Corolario las transformaciones están dadas cuando el estudiante puede establecer cuáles elementos del teorema se mantienen y cuáles cambian para el caso particular. Una evidencia de esto es el trabajo realizado por el estudiante EC6, quien escribe: "La composición de funciones se representa por la multiplicación de las matrices asociadas, en este caso $[T]_{B}^{B}$ consigo misma”. De manera similar EC4 escribe el teorema TLMA y el Corolario como un resultado directo. A continuación aparece el resultado escrito por EC6: "Por Teorema $[F \circ T]_{B}^{D}=[F]_{C}^{D} \cdot[F]_{B}^{C} ; T: U \rightarrow V ; F: V \rightarrow W$ y $B, C, D$ bases de $U, V$ y $W$. Luego en nuestro caso

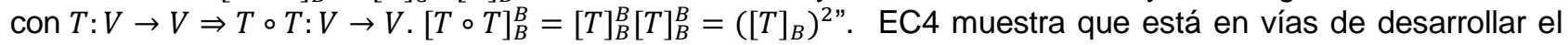
invariante, que identifica el nivel Trans del Corolario del teorema TLMA, al escribir la matriz asociada de la composición de una transformación lineal con ella misma, como una potencia.

Evidencias de la construcción del Esquema del teorema en un nivel Trans-TLMA. Mayoritariamente los estudiantes del Caso 1 al resolver la pregunta 4, muestran categóricamente la importancia del dominio y recorrido de las funciones, evidenciando así un trabajo primario en funciones que posteriormente interpretan como transformaciones lineales, hecho que sustenta la existencia del invariante de la composición de funciones. Esto último sitúa el trabajo de estos estudiantes en el nivel Trans-TLMA. En la Tabla 10 puede encontrarse evidencia del trabajo realizado por EC3.

Tabla 10: Procedimiento de EC3 en la pregunta 4

\begin{tabular}{|l|lc|}
\hline \multicolumn{1}{|c|}{$\begin{array}{l}\text { Pregunta 4 } \\
\begin{array}{l}\text { Define funciones entre espacios } \\
\text { vectoriales: Transformaciones }\end{array}\end{array}$} & $\begin{array}{l}\text { Para que } f \circ g \text { y } g \circ f \text { estén bien definidas, tienen que ser de la } \\
\text { forma } f: U \rightarrow V, g: V \rightarrow U . \text { Así, las siguientes transformaciones } \\
\text { cumplen lo anterior }\end{array}$ \\
& $f: \mathbb{R}^{3} \rightarrow \mathbb{R}^{2}$ & $g: \mathbb{R}^{2} \rightarrow \mathbb{R}^{3}$ \\
$(x, y, z) \rightarrow(x-y, 2 z)$ & $(x, y) \rightarrow(3 x, y-x, x+y)$ \\
\hline
\end{tabular}

Paso seguido define las transformaciones $f$ y $g$ demostrando que son lineales. A continuación aplica la composición de funciones sobre transformaciones lineales (Tabla 11).

Tabla 11: Evidencias de la construcción del nivel Trans-TLMA

\begin{tabular}{|c|c|}
\hline & $(f \circ g)(x, y)=f(g(x, y))$ \\
& $=f(3 x, y-x, x+y)$ \\
Pregunta 4 & $(4 x-y, 2 x+2 y)$ \\
Invariante componer transformaciones & \\
lineales como funciones & $(g \circ f)(x, y, z)=g(f(x, y, z))$ \\
& $=g(x-y, 2 z)$ \\
& $=(3 x-3 y, 2 z-x+y, x-y+2 z)$ \\
\hline
\end{tabular}

Para el ítem b. de la pregunta 4, los estudiantes proponen bases canónicas de los espacios. Este es el caso de $E C 1$, quien define las funciones $f: \mathbb{R}^{2} \rightarrow M_{2 \times 1}(\mathbb{R})$ y $g: M_{2 \times 1}(\mathbb{R}) \rightarrow \mathbb{R}^{2} \operatorname{como} f(x, y)=\left(\begin{array}{l}x \\ y\end{array}\right)$ y $g\left(\begin{array}{l}x \\ y\end{array}\right)=(y, x)$ y considerando esas formas analíticas las compone $(f \circ g)\left(\begin{array}{l}x \\ y\end{array}\right)=\left(\begin{array}{l}y \\ x\end{array}\right)$ y $(g \circ f)(x, y)=(y, x)$. Este estudiante muestra conocer la relación que establece el teorema TLMA, pues lo usa para determinar las matrices que representan las funciones $(f \circ g)$ y $(g \circ f)$, esto puede verse en la Tabla 12. 
Tabla 12: Procedimiento de EC1 en la pregunta 4.b

\begin{tabular}{|l|c|}
\hline \multirow{3}{*}{ Pregunta 4 } & {$[f \circ g]_{B}^{B}=[f]_{A}^{B}[g]_{B}^{A}=\left[\begin{array}{ll}1 & 0 \\
0 & 1\end{array}\right] \cdot\left[\begin{array}{ll}0 & 1 \\
1 & 0\end{array}\right]=\left[\begin{array}{ll}0 & 1 \\
1 & 0\end{array}\right]$} \\
& {$[g \circ f]_{A}^{A}=[g]_{B}^{A}[f]_{A}^{B}=\left[\begin{array}{ll}0 & 1 \\
1 & 0\end{array}\right] \cdot\left[\begin{array}{ll}1 & 0 \\
0 & 1\end{array}\right]=\left[\begin{array}{ll}0 & 1 \\
1 & 0\end{array}\right]$} \\
\hline
\end{tabular}

Esta forma de uso muestra que EC1 ha dado paso hacia un nivel más avanzado en la construcción del teorema TLMA, que lo sitúa en un nivel Trans-TLMA. Esto último es evidencia pura que el uso, en este caso de un teorema, se convierte en un medio de construcción conceptual de un fragmento de la matemática.

\section{CONCLUSIONES}

Los resultados de esta investigación muestran las estructuras y mecanismos mentales que modelan el aprendizaje del teorema TLMA. Entre ellas se destacaron las construcciones Objeto de la representación matricial de una transformación lineal y Proceso del vector de coordenadas. En cuanto a la caracterización de la evolución del Esquema del teorema TLMA a través de indicadores en sus niveles, el análisis de los resultados obtenidos muestra evidencias de haber logrado las siguientes estructuras y desarrollado mecanismos mentales asociados a ella:

Concepción Objeto de la Representación Matricial de una Transformación Lineal que resulta de la Asimilación del Objeto matriz por el Esquema de Transformación Lineal: Los estudiantes del Caso 2 mostraron que para dar paso a la Asimilación se deben coordinar las estructuras Procesos de vector de coordenadas y de representación matricial de la transformación lineal a través de la fijación de una base ordenada. Que permite acomodar el Esquema de transformación lineal; así el estudiante puede interpretar la matriz más allá que un arreglo rectangular, específicamente resaltar características propias de la transformación lineal como función que preserva combinaciones lineales. Por ejemplo determinando la dimensión de los espacios vectoriales sobre los cuales está definida dicha función.

Construcción del Esquema del teorema nivel Intra-TLMA: Estudiantes del Caso 1 evidenciaron relacionar la representación matricial de la composición de funciones con la multiplicación de las representaciones matriciales de las funciones componentes, a través de un argumento matemático situado específicamente en bases canónicas de los espacios vectoriales involucrados.

Construcción del Esquema del teorema nivel Inter-TLMA: El estado de evolución de los estudiantes del Caso 1 del nivel Intra al Inter del Esquema del teorema TLMA, está sustentado en las transformaciones de las coordenadas de las imágenes a través de una base $\mathrm{B}$, que no es necesariamente la base canónica. Estas transformaciones les permiten construir la representación matricial, a partir de transponer los vectores coordenadas de la imágenes bajo la transformación.

Construcción del Esquema del teorema nivel Trans-TLMA: El invariante que caracteriza el trabajo de los estudiantes en este nivel, está dado por la composición de transformaciones lineales como funciones. Específicamente los estudiantes del Caso 1 inician la composición situada en el dominio de las funciones definidas en el campo de los reales, que posteriormente trasladan a un dominio de las transformaciones lineales como funciones definidas entre espacios vectoriales.

El trabajo evidenciado por los estudiantes señala la importancia de la construcción del teorema TLMA para establecer conexiones entre los elementos descritos en la Descomposición Genética. Referente al Corolario, éste es incorporado por los estudiantes del Caso 2 como una forma particular del teorema TLMA. El análisis de los datos muestra que los estudiantes pueden pensar en la composición de funciones como la potencia de una matriz. Las transformaciones en este caso referentes a qué permanece del teorema TLMA y qué varía respecto al corolario, están definidas por la composición de una transformación lineal con sí misma y el producto matricial entre matrices cuadradas. Dependiendo del uso que un estudiante haga del teorema TLMA podrá elegir una base (canónica, ortogonal u ortonormal) para determinar la forma matricial de una transformación lineal que mejor se acomode a las condiciones del contexto; por tanto este uso establece una forma de construcción conceptual. Como puede verse en la Tabla 13, una transformación lineal puede definirse en lenguaje natural y ser representada a través de una matriz que genera un efecto sobre una figura geométrica.

Por ejemplo, consideremos las transformaciones lineales, $S: \mathbb{R}^{2} \rightarrow \mathbb{R}^{2}, S(x, y)=(2 x, y) \quad$ y $T: \mathbb{R}^{2} \rightarrow \mathbb{R}^{2}$, $T(x, y)=(y,-x)$. En un contexto algebraico la Transformación $T \circ S$ es $T(S(x, y))=T(2 x, y)=(y,-2 x)$. 
Ahora de acuerdo al Teorema TLMA la matriz de composición de $T \circ S$, esta dada por: $\left(\begin{array}{cc}2 & 0 \\ 0 & 1\end{array}\right)\left(\begin{array}{cc}0 & -1 \\ 1 & 0\end{array}\right)=$ $\left(\begin{array}{cc}0 & -2 \\ 1 & 0\end{array}\right)$. Cuyo efecto geométrico se puede visualizar el la Figura 4.

Tabla 13: Transformaciones lineales representadas de forma matricial y geométrica

\begin{tabular}{|c|c|c|}
\hline Transformación lineal & $\begin{array}{c}\text { Representación matricial en las } \\
\text { bases canónicas }\end{array}$ & $\begin{array}{c}\text { Efecto sobre el cuadrado } \\
\text { unitario }\end{array}$ \\
\hline S es una Expansión horizontal & $N=\left(\begin{array}{ll}k & 0 \\
0 & 1\end{array}\right), \quad k=2$ & 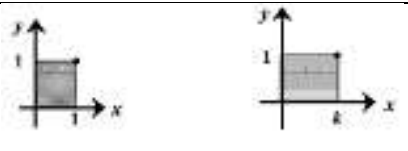 \\
\hline$T$ es una Rotación en 90ํำ & $M=\left(\begin{array}{cc}0 & -1 \\
1 & 0\end{array}\right)$ & 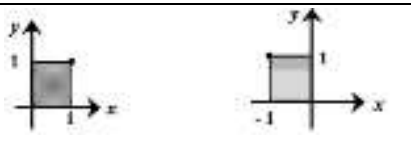 \\
\hline
\end{tabular}

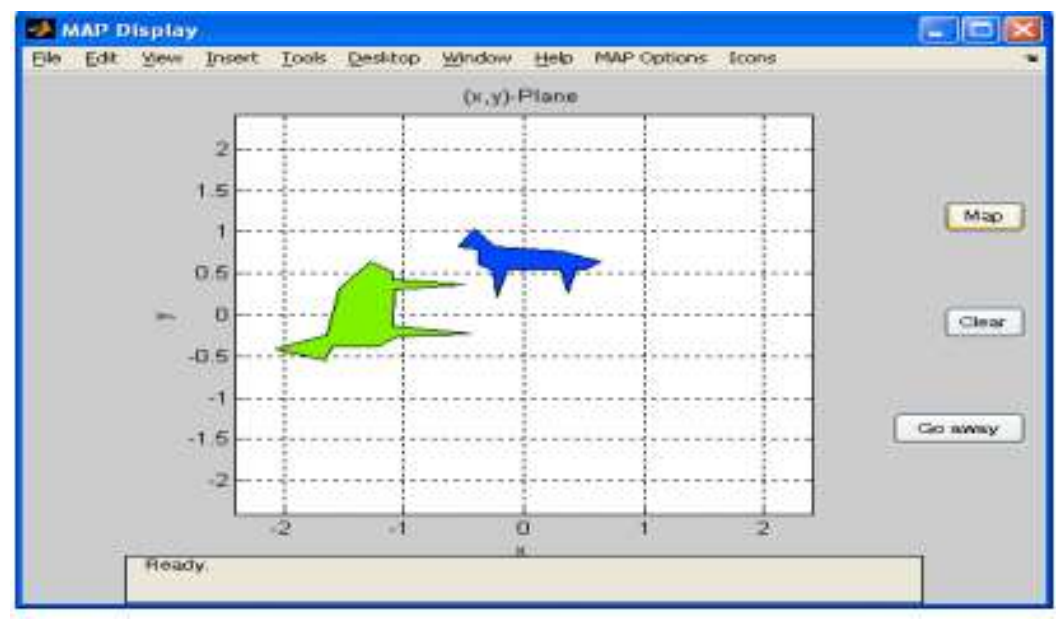

Fig. 4: Efecto geométrico de la transformación lineal $T \circ S$

Sin embargo un estudiante podría querer visualizar qué sucede con el efecto geométrico al aplicar primero la Rotación seguida de la matriz de expansión horizontal, esto es $S \circ T, S(T(x, y))=S(y,-x)=(2 y,-x)$, cuya matriz de composición por el teorema TLMA está dada por:

$$
\left(\begin{array}{cc}
0 & -1 \\
1 & 0
\end{array}\right)\left(\begin{array}{ll}
2 & 0 \\
0 & 1
\end{array}\right)=\left(\begin{array}{cc}
0 & -1 \\
2 & 0
\end{array}\right)
$$

Cuyo efecto geométrico se puede visualizar en la Figura 5.

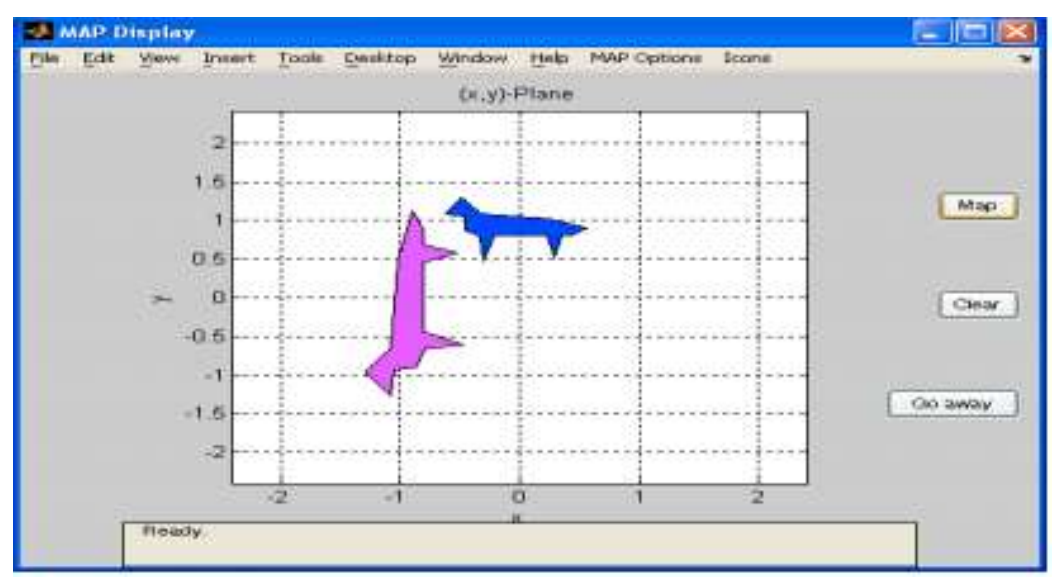

Fig. 5: Efecto geométrico de la transformación lineal $S \circ T$ 
Este ejemplo particular es importante porque demuestra a través de un contexto algebraico, matricial y geométrico que la composición de transformaciones lineales no es conmutativa (ver Tabla 14), y el estudiante puede decidir cuál de estos contextos y sus condiciones ilustra mejor para él la no conmutatividad de esta operación.

Tabla 14: Contextos algebraico, matricial y geométrico para la composición de transformaciones lineales dadas

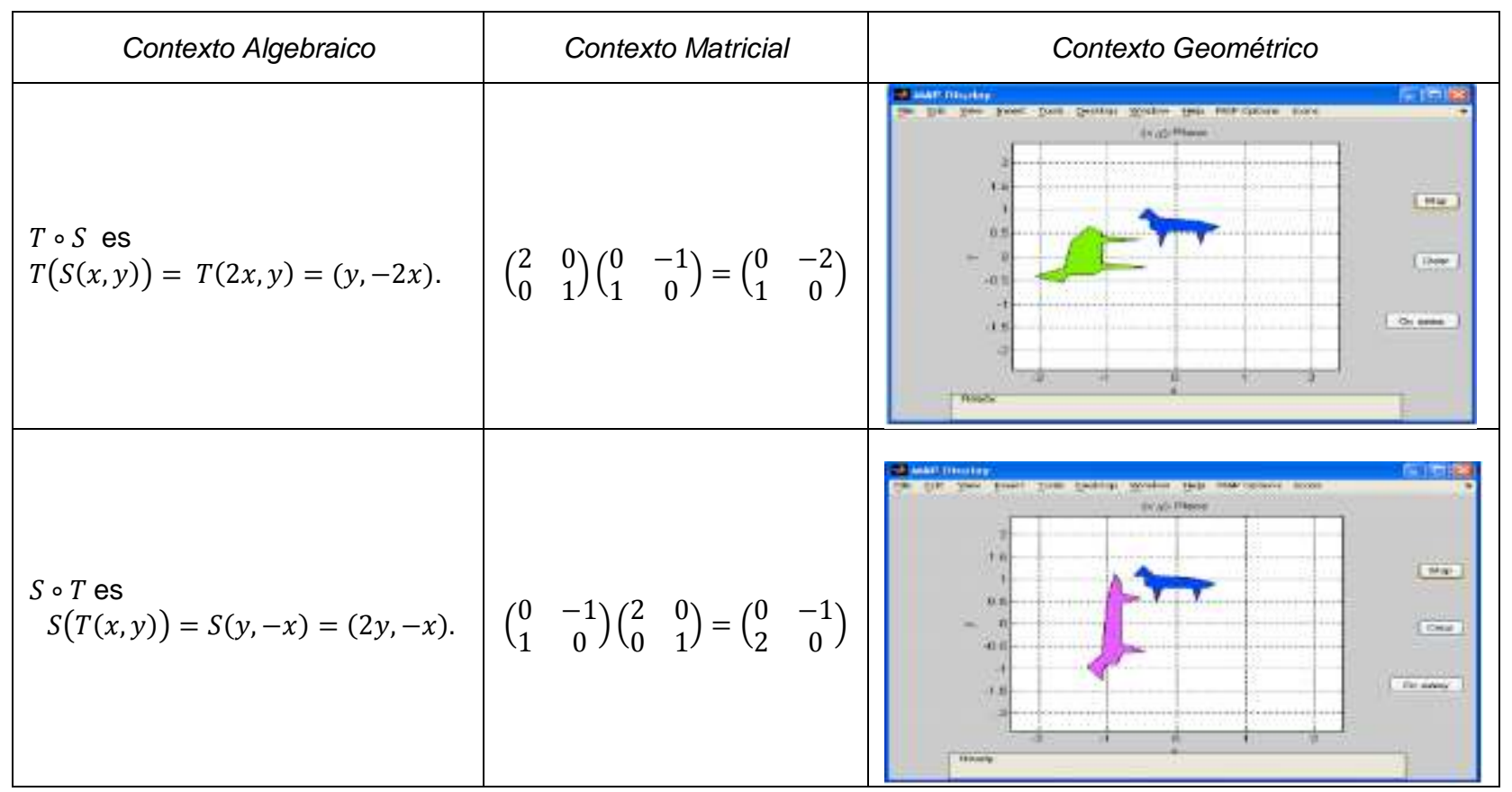

Las evidencias obtenidas dan cuenta que las dificultades de los estudiantes para comprender el teorema TLMA están determinadas por el mecanismo de Asimilación. El trabajo de los estudiantes del Caso 2, evidencia la aplicación mecánica de un algoritmo para calcular la representación matricial de una transformación lineal dada en su forma analítica. Esto se refleja en sus procedimientos ya que no fijan las bases de los espacios sobre los que se definen la función para determinar la representación matricial y/o no pueden obtener información de la transformación lineal en su forma analítica a partir de la matriz que la representa. Por ejemplo determinando las dimensiones de los espacios sobre los que se define la función.

Desde la matemática el análisis de los resultados muestra que la no construcción de la base como concepto unificador de: coordenadas, transformaciones lineales y composición de funciones, imposibilita la evolución del teorema TLMA y su Corolario. Esta dificultad implica que no se podrán construir relaciones, transformaciones e invariantes que evidencian la evolución del Esquema del teorema TLMA. En consecuencia el aprendizaje del teorema TLMA estará limitado al dominio de tópicos matemáticos particulares. Por ejemplo, bases canónicas y transformaciones lineales definidas en $\mathbb{R}^{n}$ dejando fuera todo un mundo de tópicos matemáticos por explorar, donde la elección de una base pude dar lugar al uso más 0 menos efectivo del teorema TLMA.

En general podemos decir que las estructuras previstas en la Descomposición Genética que aparecen en el trabajo de los estudiantes, son fundamentalmente: una estructura Objeto de la representación matricial de una transformación lineal y Objeto de la composición de funciones. Específicamente la importancia del reconocimiento de las bases para la representación matricial y de la dimensión para el orden la matriz, estarían influyendo fuertemente en la construcción de relaciones, transformaciones e invariantes. Estos datos sustentan que la Descomposición Genética diseñada modela las estructuras necesarias para el aprendizaje del teorema TLMA.

Con este estudio se propone a la comunidad de profesores e investigadores universitarios una primera respuesta a la pregunta de investigación planteada, acerca de las estructuras y mecanismos mentales que modelan la construcción del teorema TLMA. Así como también una Descomposición Genética que incluye las estructuras previas que dan paso a la construcción del teorema. Esto representa una herramienta para que los profesores universitarios diseñen propuestas de aprendizaje para el teorema TLMA y otros que pueden propiciar el desarrollo del pensamiento abstracto en álgebra lineal. Sin embargo este estudio va más allá de la validación de una Descomposición genética. Entre las contribuciones que la investigación hace a la literatura se pueden señalar el estudio de indicadores para interpretar los niveles de los esquemas Intra, inter y Trans del teorema TLMA. Esto último con base en la asimilación del Objeto matriz por el esquema de transformación lineal a través de la fijación de una base ordenada. 
Este estudio proporciona nueva evidencia de que el uso de los teoremas y su análisis desde la teoría APOE permite determinar las construcciones que subyacen a las dificultades de los estudiantes y a sus estrategias cuando desarrollan una actividad matemática en álgebra lineal y fuera de ella. Se sugiere para futuras investigaciones aplicar el ciclo de investigación con base en el camino cognitivo descrito, teniendo en cuenta en la toma y análisis de datos, el diseño y desarrollo de entrevistas didácticas que permitan conocer con mayor profundidad, los indicadores de interpretación de los niveles del Esquema del teorema TLMA.

\section{AGRADECIMIENTOS}

Este trabajo ha sido subvencionado parcialmente por el proyecto FONDECYT 1140801. La primera autora agradece el apoyo brindado por el Programa de Movilidad 2016 de la Vicerrectoría de Investigación y Extensión de la Universidad Industrial de Santander VIE-UIS. Los autores agradecen la buena disposición a los participantes en la investigación.

\section{REFERENCIAS}

Abramovitz, B., Berezina, M., Berman, A. y Shvartsman, L., How to understand a theorem? International Journal of Mathematical Education in Science and Technology, 40(15), 577-586 (2009)

Arnon, I., Cottrill, J., Dubinsky, E., Oktaç, A., Roa-Fuentes, S., Trigueros, M. y Weller, K., APOS Theory. A Framework for research and curriculum development in mathematics education, (12-235), Springer, New York, U.S.A. (2014)

Asiala, M., Anne, B., DeVries, D.J., Dubinsky, E., Mathews, D. y Thomas, K., Framework for Research and Curriculum Development in Undergraduate Mathematics Education. Research in Collegiate Mathematics Education II, CBMS Issues in Mathematics Education, American Mathematical Society, pp. 1-32, Michigan, U.S.A. (1996)

Asiala, M., Brown, A., Kleiman, J. y Mathews, D., The development of students' understanding of permutations and symmetries, International, Journal of Computers for Mathematical Learning, 3(1), 13 - 43 (1998)

Balacheff, N., Processus de preuve et situations de validation, Educational Studies in Mathematics, 18, 147$176(1987)$

Carlson, D., Johnson, C., Lay, D., Porter, A., Watkins, A. y Watkins, W., Resources for Teaching Linear Algebra, Mathematical Association of America Notes, 42, pp. 107-126, Washington, U.S.A. (1997)

Carlson, M. y Oehrtman, M., Key aspects of knowing and learning the concept of function, Research Sampler Series, 9 [The Mathematical Association of America Notes Online] (2015)

Dorier, J.L., Epistemological analysis of the genesis of the theory of vector spaces. On the Teaching of Linear Algebra, Kluwer Academic Publishers, pp. 3-81, The Netherlands (2000)

Dubinsky, E., Reflective abstraction in advanced mathematical thinking, Advanced mathematical thinking, Springer, pp. 95-123, Dordrecht, Springer Netherlands (1991)

Dubinsky, E. y McDonald, M.A., APOS: A constructivist theory of learning in undergraduate mathematics education research, The teaching and learning of mathematics at university level, pp. 275-282, Springer Netherlands (2001)

Figueroa, G. y Fierro, R., Álgebra Lineal, Instituto de Matemáticas PUCV, Valparaíso, Chile (1999)

Hoffman, K., y Kunze, R., Álgebra lineal, Prentince-Hall, Ciudad de México, México (1993)

Kú, D., Trigueros, M. y Oktaç, A., Comprensión del concepto de base de un espacio vectorial desde el punto de vista de la Teoría APOE, Educación Matemática, 20(2), 65-89 (2008)

Mena, A., Morales, A., y Parraguez, M., Mental Constructions for the Group Isomorphism Theorem, Mathematics Education, 11(2), 377-393 (2016)

McDonald, M.A., Mathews, D. y Strobel, K., Understanding sequences: A tale of two objects, Research in Collegiate Mathematics Education, 4, 77-102 (2000) 
Parraguez, M., y Oktaç, A., Desarrollo de un esquema del concepto espacio vectorial, Paradigma, 33(1), 103-134 (2012)

Parraguez, M., Mental constructions and mechanisms for the use of the basic concepts of linear algebra. Proyecto Fondecyt No 1140801. Chile: CONICYT (2014)

Parraguez, M., Construcciones y mecanismos mentales para el uso de los conceptos básicos del álgebra lineal. En C. Vásquez, H. Rivas, N. Pincheira, F. Rojas, H. Solar, E. Chandía y M. Parraguez (Eds.). Jornadas Nacionales de Educación Matemática XIX. SOCHIEM, Villarrica, Chile, 48-56 (2015)

Parraguez, M., Lezama. J. y Jiménez, R., Estructuras mentales para modelar el aprendizaje del teorema de cambio de base de vectores, Enseñanza de las Ciencias, 34(2), 129 - 150 (2016)

Roa-Fuentes, S. y Oktaç, A., Validación de una descomposición genética de transformación lineal: Un análisis refinado por la aplicación del ciclo de investigación de APOE, Revista Latinoamericana de Investigación en Matemática Educativa, 15 (2), 199-232 (2012)

Salgado, H. y Trigueros, M., Teaching eigenvalues and eigenvectors using models and APOS Theory, The Journal of Mathematical Behavior, 39, 100-120 (2015)

Stake, R., Investigación con estudio de casos, $5^{\text {a }}$ Ed., Morata, Madrid, España (2010)

Trigueros, M. y Martínez-Planell, R., Geometrical representations in the learning of two-variable functions, Educational Studies in Mathematics, 73(1), 3-19 (2010)

Trigueros, M., Vínculo entre la modelación y el uso de representaciones en la comprensión de los conceptos de ecuación diferencial de primer orden y de solución, Educación Matemática, 207-226 (2014)

Uhlig, F., A New Unified, Balanced and Conceptual Approach to Teaching Linear Algebra, Linear Algebra and Its Applications, 36(1), 147-159 (2003)

Valdivia, C. y Parraguez, M., Un Modelo Cognitivo para la Comprensión Profunda de la Regla de la Cadena, Paradigma, 35(2), 146-176 (2015)

Vásquez, C. y Parraguez, M., Construcciones mentales para el aprendizaje del concepto de probabilidad: Un estudio de caso, Revista Educación Matemática, 26(3), 37-74 (2014)

Villabona, D. y Roa-Fuentes, S., Procesos iterativos infinitos y objetos trascendentes: un modelo de construcción del infinito matemático desde la teoría APOE, Educación Matemática, 28(2), 119-150 (2016)

Weller, K., Montgomery, A., Clark, J., Cottrill, J., Trigueros, M. y Arnon, I., Learning Linear Algebra with ISETL. Disponible en: https://goo.gl/Qx4qiZ. Preliminary Version 3, July 31 (2002) 